\title{
DiE ALte GESCHICHTE IN WIEN UND DIE AKTUELLE ÖSTERREICHISCHE FORSCHUNG ZUM ANTIKEN BARBARICUM
}

\author{
Kurt Tomaschitz \& Igor Lisový
}

\begin{abstract}
The paper deals with the question of research of Classical Antiquity in Austria, first of all at the University of Vienna, and also problems of „barbaricum“ in the contemporary Austrian historiography. The emphasis is put on the role of G. Dobesch, Professor of the University of Vienna.

Zum 65. Geburtstag von Gerhard Dobesch, O. Prof. für Römische Geschichte, Altertumskunde und Epigraphik an der Universität Wien
\end{abstract}

In der Geschichte des Instituts für Alte Geschichte und Altertumskunde, Papyrologie und Epigraphik der Wiener Universität ${ }^{1}$ sind zwei Daten von besonderer Bedeutung: das Jahr 1876, als das Archäologisch-epigraphische Seminar gegründet wurde, das schon vor dem ersten Weltkrieg zu einem der bekanntesten europäischen Zentren für die Erforschung der Geschichte und der Kultur des Altertums wurde, und das Jahr 1984, als aus dieser Institution zwei neue entstanden - das Institut für Klassische Archäologie und das Institut für Alte Geschichte, Altertumskunde und Epigraphik.

Zur Entwicklung der Altertumswissenschaften, vor allem der Klassischen Archäologie, trug maßgeblich Alexander Conze († 1914) bei, der besonderen Wert auf die Kenntnis nicht nur der archäologischen, sondern auch der schriftlichen Quellen legte; seit dem Jahre 1869 war er Inhaber des Lehrstuhls für Klassische Archäologie ${ }^{2}$. Seit 1876 führte er gemeinsam mit Professor Otto Hirschfeld-zuvor Professor der Universitäten in Göttingen und in Prag und Schüler Theodor Mommsens - das Archäologisch-epigraphische Seminar und ein Jahr später begann er die "Archäologisch-epigraphischen Mitteilungen aus Österreich-Ungarn“ herauszugeben, die nach dem Jahr 1898 durch die "Jahreshefte des Österreichischen Archäologischen Instituts“ ersetzt wurden.

Als A. Conze nach Berlin ging, um die Direktion des dortigen Skulpturenmuseums zu übernehmen, wurde Otto Benndorf sein Nachfolger. Er unternahm eine erfolgreiche Expedition nach Kleinasien, von wo er die Reliefs des Heroons von Gjölbaschi-Trysa nach Wien mitbrachte. Im Jahre 1895 begann O. Benndorf mit den Ausgrabungen in Ephesos, die seit damals einen zentrales Projekt der österreichischen Archäologie bilden, und 1898 gründete er das Österreichische Archäologische Institut (ÖAI).

\footnotetext{
1 So lautet seit Anfang 2005 die offizielle Bezeichnung, nachdem im April 2004 Bernhard Palme zum Professor für Alte Geschichte und Papyrologie bestellt worden ist.

${ }^{2}$ Kenner 1977.
} 
Die nächsten von den Wiener Professoren und Wissenschaftlern, die bedeutende Beiträge zur Entwicklung der Altertumswissenschaften leisteten, waren Emil Reisch, Arnold Schober, Emanuel Löwy, Camillo Praschniker und Otto Walter. Emil Reischs Arbeiten über das antike Theater und über Weihgeschenke werden in wissenschaftlichen Kreisen bis heute hoch geschätzt ${ }^{3}$. Arnold Schober († 1959) verfaßte ein grundlegendes Sammelwerk über die Sepulkraldenkmäler von Noricum und Pannonien und veröffentlichte auch ein wertvolles Buch zur Kunst von Pergamon ${ }^{4}$. Camillo Praschniker, vormals Professor der deutschen Universität in Prag, erforschte die Metopen des Parthenon und reorganisierte die schon seit Conzes Zeiten bestehende Archäologische Sammlung, bestehend aus Gipsabgüssen antiker Bildwerke.

Von den Professoren, die sich mit der Alten Geschichte beschäftigten, ragten der schon erwähnte Otto Hirschfeld, der den Beginn der Grabungen in Carnuntum mit initiiert hat, und sein Nachfolger Eugen Bormann hervor ${ }^{5}$. Weitere berühmte österreichische Historiker in diesem Bereich waren Wilhelm Kubitschek aus Pressburg und Adolf Wilhelm (geboren 1864 in Böhmen in einer österreichischen Familie). Mit den Namen Otto Hirschfeld, Eugen Bormann, Wilhelm Kubitschek und auch Emil Szanto und Adolf Wilhelm beginnt die Blütezeit der Epigraphik an Wiener Universität, die zu einem Zentrum der europäischen Forschung in diesem Bereich wird.

Von den nächsten Altertumsforschern an der Wiener Universität sind Rudolf Egger (geboren 1882) und Josef Keil (geboren 1878) hervorzuheben. Der erste beschäftigte sich mit der Geschichte Österreichs in römischer Zeit und mit der Spätantike, er war aber auch ein begabter Epigraphiker. J. Keil hatte großes Interesse an Religionsgeschichte, während er als Direktor des ÖAI in den Jahren 1926-35 die Ruinen von Ephesos erforschte.

In der Nachkriegszeit ist unter den vornehmen österreichischen Forschern der alten Geschichte die Persönlichkeit von Artur Betz, dem Schüler von Egger (geboren 1905), zu nennen, der 1956 eine wertvolle Geschichte der Austria Romana vorlegte. Im selben Jahr wurde das Archäologisch-epigraphische Seminar in Institut für Alte Geschichte, Archäologie und Epigraphik umbenannt. Dadurch wurden die Voraussetzungen für die Entwicklung der Wiener historischen Schule geschaffen, an deren Formierung die bekannten Forscher Rudolf Egger, Artur Betz, Fritz Schachermeyr, Ernst Kirsten, Gerhard Dobesch und Ekkehard Weber beteiligt waren. 1983 übernahm Peter Siewert von Kirsten den Lehrstuhl für Griechische Geschichte, Altertumskunde und Epigraphik und von 1994-2001 bekleidete Luciana Aigner-Foresti eine Professur für Etruskologie und italische Altertumskunde, in dieser Form die einzige im deutschen Sprachraum.

Gerhard Dobesch (geboren 1939), Schüler von Schachermeyr und seit 1963 als Assistent am Wiener Institut tätig6, entwickelte früh ein besonderes Interesse an Caesar, über den er 1966 seine erste Monographie veröffentlichte ${ }^{7}$. Seine Gedanken setzten eine rege Diskussion in Gang und erfuhren auch Widerspruch (z.B. H. Gesche,

\footnotetext{
${ }^{3}$ Dörpfeld \& Reisch 1896; Reisch 1890.

${ }^{4}$ Schober 1923 und 1951.

${ }^{5}$ Dobesch 1977.

${ }^{6}$ Für eine Würdigung seiner Persönlichkeit und seines Werks vgl. Franke 2001; Lisový \& Tomaschitz 2003-2004.

${ }^{7}$ Dobesch 1966; zu ihrem Inhalt, ebenso zum Inhalt der anderen Monographien vide Dobesch 2004b.
} 
Die Vergottung Caesars, 1968), trotzdem blieb Dobeschs Meinung zur Problematik anerkannt und wird bis heute rezipiert. 1967 habilitierte sich Dobesch mit der Monographie Der panhellenische Gedanke im 4. Jh. v. Chr. und der "Philippos" des Isokrates. Untersuchungen zum korinthischen Bund I. (1968 in Wien erschienen) zum Universitätsdozenten im Bereich der Alten Geschichte am oben erwähnten Institut. Im gleichen Jahr wurde er korrespondierendes Mitglied des ÖAI.

Nachdem er von 1973-76 als Ordentlicher Universitätsprofessor der Alten Geschichte und Altertumskunde an der Karl Franzens-Universität in Graz gelehrt hatte und im Jahre 1976 zum Ordinarius für Römische Geschichte, Altertumskunde und Epigraphik an der Universität Wien berufen worden war, erschien 1980 seine Monographie über die Kelten in Österreich, in der er die Frage der Beziehungen zwischen den Kelten und Rom im zweiten Jahrhundert v. Chr. untersuchte ${ }^{8}$. Im selben Jahr wurde er korrespondierendes Mitglied und 1984 wirkliches Mitglied der Österreichischen Akademie der Wissenschaften. Im Jahre 1988 erfolgte seine Wahl zum Vorsitzenden der Kleinasiatischen Kommission der Österreichischen Akademie der Wissenschaften und 1998 wurde er wirkliches Mitglied des ÖAI und auch Socio Straniero dell'Istituto Nazionale di Studi Etruschi e Italici.

Seine Forschungsschwerpunkte sind: a) der gesamte Bereich der Alten Geschichte und der antiken Kultur- und Geistesgeschichte in ganzheitlicher Schau aus universalhistorischer Perspektive, b) die Phase des Übergangs von der römischen Republik zur Kaiserzeit mit besonderem Interesse an Caesar, Cicero und Augustus, c) das keltische und germanische „Barbaricum“, vor allem die Struktur der „barbarischen" Politik und Geschichte, d) Probleme der Spätantike. Die praktische Umsetzung dieser Interessen stellen seine Monographien über Kelten und Germanen ${ }^{9}$ sowie über das europäische „Barbaricum“ und die Zone der Mittelmeerkultur nach Poseidonios $^{10}$ dar. Dieses letztgenannte Buch besteht aus 41 kurzen Kapiteln, in denen der Autor dem Leser die Interpretation des "Barbaricums" in der modernen Wissenschaft (S. 9-11) und sein Verständnis der antiken Autoren, z.B. Timaios, Hekataios von Abdera, Apollonios von Rhodos und Diodoros (S. 35-37, 70-85), nahebringt. Die größte Aufmerksamkeit widmet er den Kelten und den Germanen im Prisma der Interpretation von Poseidonios von Apameia, den er für einen Autor hält, der sich um einen gesamtheitlichen Blick auf das europäische „Barbaricum“ bemüht (S. 5964, 91-99, 107-109). Dobesch geht es in diesem Werk vor allem um die Bedeutung und Anwendung des Wortes „Barbaren“. Er gelangt zum Schluss (S. 15-16), dass damit nicht Unkultivierte, sondern Fremdkultivierte bezeichnet sind. Deswegen ist es nötig, unseren Barbarenbegriff zu ändern. Bei den Völkern des Nordens und Ostens spüren wir etwas Besonderes, das sich aber schwer konkretisieren läßt, weil wir weder die Mythologie noch die Poesie dieser Nationen kennen, die uns ihre Weltsicht vermitteln könnten. Das Barbaricum bildet wie die Städtezone des Mittelmeeres und Vorderasiens eine eigene Oikumene und trotz vielfacher Querbeziehungen lebten diese getrennten Welten, jede in sich wiederum alles eher als einheitlich, weitgehend nebeneinander her.

\footnotetext{
${ }^{8}$ Dobesch 1980.

${ }^{9}$ Vgl. Dobesch 1994.

${ }^{10}$ Dobesch 1995 (Rezension: Burian 1996). Diese Monographie ist auch in tschechischer Sprache publiziert, siehe Dobesch 2004a.
} 
Die Frage der römischen Beziehungen zur sog. barbarischen Welt, die in der europäischen historischen Wissenschaft in der Nachkriegsepoche hervortrat, beschäftigt heute vor allem die Forscher jener europäischen Länder, die mit den Römern entweder als Provinzen oder als bestimmte Kontaktzonen in Berührung kamen. Diese Problematik zog auch die Aufmerksamkeit der tschechischen, slowakischen (z.B. J. Dobiáš, J. Burian, P. Oliva, J. Bouzek, V. Sakař, K. Kurz) und der österreichischen Forscher auf sich, unter denen Prof. Gerhard Dobesch eine der ersten Stellen einnimmt. Er hat mit seinen Arbeiten zum antiken europäischen Barbaricum, die neben den drei erwähnten Monographien auch zahlreiche Aufsätze umfassen, einen besonders gewichtigen Beitrag zur Erforschung dieses Themenkomplexes geleistet. Sein Interesse an den Ländern und Völkern des alten Europa, die außerhalb der mediterranen Zone bzw. der römischen Reichsgrenzen liegen, wird in der zeitgenössischen österreichischen Geschichtsforschung aber von vielen Vertretern der Alten Geschichte und ihrer Nachbarfächer geteilt. Im Folgenden soll ein Überblick über die aktuelle Situation gegeben werden, der angesichts der gebotenen Kürze natürlich keine Vollständigkeit beanspruchen kann. Auch bei den bibliographischen Angaben musste, vor allem bei den nicht monographischen Titeln, eine Auswahl getroffen werden.

Im Bereich der Alten Geschichte wird zu dem Thema an allen UniversitätsStandorten, an denen das Fach vertreten ist-neben Wien sind das Graz, Salzburg, Innsbruck und Klagenfurt-geforscht. Am Institut für Alte Geschichte und Altorientalistik in Innsbruck liegt ein allgemeiner Schwerpunkt auf den Beziehungen der griechisch-römischen Welt zu den anderen Kulturkreisen der Antike, im Besonderen zu den orientalischen Kulturen. In diesem Rahmen hat die Beschäftigung von Reinhold Bichler mit Herodot und seinen ethnographischen Aussagen reiche Ergebnisse gebracht ${ }^{11}$, die unser Verständnis dieses für die antike Ethnographie so wichtigen Autors wesentlich gefördert haben. Von Bichlers Schülern hat Wido Sieberer mit seiner Dissertation über Herodots Bild von Europa eine grundlegende Arbeit zum betrachteten Themenkomplex publiziert ${ }^{12}$, während Robert Rollinger sich neben der Beschäftigung mit Herodot auch die Geschichte der Austria Romana angelegen sein läßt ${ }^{13}$. Zu speziellen Problemen des Alpenraums in vorrömischer Zeit hat sich auch Peter Haider geäußert ${ }^{14}$.

Am Institut für Alte Geschichte und Altertumskunde der Universität Graz hat sich Klaus Tausend bereits in seiner Dissertation mit der Darstellung der Hunnen bei den spätantiken Historikern beschäftigt und diesem Volk noch weitere Beiträge gewidmet, sich darüber hinaus aber auch den Germanen als Nachbarn des Imperium Romanum zugewandt ${ }^{15}$. Manfred Hainzmann wiederum hat aus seiner intensiven Beschäftigung mit dem Inschriftenmaterial des Alpen- und Donauraumes heraus zunächst über die Kelten Österreichs geforscht und in jüngster Zeit die Koordination

\footnotetext{
${ }^{11}$ Bichler 2000a und 2000b.

12 Sieberer 1995.

${ }^{13}$ Bichler \& Rollinger 2001; Rollinger 1995, 1998 und 2001.

${ }^{14}$ Haider 1993 und 2000.

15 Tausend 1984, 1985/86, 1987, 1988, 1991, 1997, 1999a und 1999b.
} 
eines großen internationalen und interdisziplinären Forschungsprojekts $\mathrm{zu}$ den Fontes Epigraphici Religionis Celticae Antiquae, kurz FERCAN genannt, übernommen ${ }^{16}$.

Am Institut für Alte Geschichte, Altertumskunde und Mykenologie in Salzburg ist Herbert Graßl hervorzuheben, der zur Frühgeschichte des Ostalpenraumes eine Reihe von Arbeiten veröffentlicht hat ${ }^{17}$, und in Klagenfurt, wo die Alte Geschichte im Rahmen des dortigen Instituts für Geschichte vertreten ist, legt Karl Strobel einen Schwerpunkt seiner aktuellen Forschungsarbeit auf das antike Keltentum, wobei das Hauptaugenmerk wohl auf den kleinasiatischen Galatern liegt, das europäische Keltentum deswegen aber keineswegs vernachlässigt wird ${ }^{18}$. Auch seine Monographien über die Beziehungen des Römischen Reiches zu den Rheingermanen und den Dakern sind an dieser Stelle zu nennen ${ }^{19}$.

In Wien, um wieder hierher zurückzukehren, hat Gerhard Dobesch nicht nur selbst über das Barbaricum geforscht, sondern auch einschlägige Themen von Schülern bearbeiten lassen. Kurt Tomaschitz hat die antiken literarischen Testimonien zu den Wanderungen der Kelten zusammengestellt und kommentiert ${ }^{20}$, Andreas Hofeneder hat in seiner Dissertation einen ersten Teil der Zeugnisse zur keltischen Religion kommentiert vorgelegt ${ }^{21}$ und arbeitet derzeit im Rahmen des oben erwähnten FERCANProjektes am zweiten Teil dieses Vorhabens, das die alte, in vieler Hinsicht überholte Sammlung von Zwicker ersetzen wird.

Über das europäische Barbaricum wird in Wien auch bei den Nachbarfächern der Alten Geschichte, bei der Ur- und Frühgeschichte und der Frühmittelalterforschung, intensiv gearbeitet und publiziert. Auf der prähistorischen Seite sind für die jüngere Eisenzeit, die große Epoche der Kelten, vor allem die Arbeiten von Otto $\mathrm{H}$. Urban zu nennen ${ }^{22}$, für die Kaiserzeit die Untersuchungen von Alois Stuppner zu den Germanen als Nachbarn des Imperium Romanum an der mittleren Donau ${ }^{23}$ und für die Völkerwanderungszeit die Schriften von Herwig Friesinger ${ }^{24}$ und Falko Daim $^{25}$. Letztere haben dabei z.T. intensiv mit der Mediävisten-Schule von Herwig Wolfram kooperiert ${ }^{26}$, dem es mit seiner richtungsweisenden Monographie über die Goten sogar gelungen ist, über das Fachpublikum hinaus eine breitere Öffentlichkeit anzusprechen $^{27}$. Der langjährige Leiter des Instituts für Österreichische Geschichtsforschung hat zur Geschichte der Völkerwanderungszeit darüber hinaus noch viele Arbeiten veröffentlicht ${ }^{28}$ und seinem anregenden Vorbild verdankt sich mittlerweile

\footnotetext{
${ }^{16}$ Hainzmann 1994, 1996, 1998, 2001a, 2001b und 2002.

17 Graßl 1998, 2000 und 2001.

18 Strobel 1996 und 2003.

${ }^{19}$ Strobel 1984 und 1989.

20 Tomaschitz 2002.

${ }^{21}$ Hofeneder 2005.

22 Urban 1994, 1995 und 2003.

${ }_{23}$ Stuppner 1994, 1995 und 1997.

${ }^{24}$ Friesinger 1994; Friesinger \& Adler 1979; Friesinger \& Vacha 1987 und 1988.

${ }^{25}$ Daim \& Bálint 1989; Daim \& Andrási 2000.

${ }^{26}$ Wolfram \& Friesinger 1985; Wolfram \& Daim 1980.

27 Wolfram 2001.

${ }^{28}$ Etwa, um nur zwei weitere Monographien zu nennen, Wolfram 1987 und 1990.
} 
eine lange Reihe substantieller Beiträge seitens seiner Schüler bzw. Mitarbeiter Walter Pohl29, Georg Scheibelreiter ${ }^{30}$ und Andreas Schwarcz ${ }^{31}$.

An der Universität Wien wird das Barbaricum freilich nicht nur von historischarchäologischer Seite betrachtet, sondern auch von den Sprachwissenschaften her erforscht. Der Germanist Helmut Birkhan, der schon in seiner Habilitationsschrift keltisch-germanische Sprachkontakte untersucht hat ${ }^{32}$, hat hier mit seiner überaus erfolgreichen Monographie über die Kelten ${ }^{33}$ eine Entwicklung in Gang gesetzt, die dann zur Einführung eines Studiengangs „Keltologie“ und zu einer Schwerpunktsetzung auf keltische Sprachen am Wiener Institut für Indogermanistik geführt hat.

Diese kurze und selektive Aufzählung kann, wie schon eingangs gesagt, keine Vollständigkeit beanspruchen, da hier z.B. die für das Thema natürlich ebenfalls relevante provinzialrömische Archäologie außer Betracht geblieben ist. Sie sollte aber eine Vorstellung davon vermitteln, welch großes Interesse dem antiken Barbaricum als Forschungsobjekt von Seiten aller involvierten Disziplinen entgegengebracht wird.

Dr. Kurt Tomaschitz

Institut für Alte Geschichte und Altertumskunde, Papyrologie und Epigraphik

Universität Wien

E-Mail: kurt.tomaschitz@univie.ac.at

Dr. Igor Lisový

Historický ústav Jihočeské univerzity

Ceské Budejovice (Budweis)

E-Mail: lisovy@hu.jcu.cz

\section{Bibliographie}

Bichler, Reinhold (2000a) Herodots Welt: der Aufbau der Historie am Bild der fremden Länder und Völker, ihrer Zivilisation und ihrer Geschichte. Mit Anlagen von Dieter Feil und Wido Sieberer. Berlin: Akademie-Verlag.

Bichler, Reinhold (2000b) ,Herodots Frauenbild und seine Vorstellung über die Sexualsitten der Völker.' - Rollinger, R.; Ulf, Ch. (Hrsg.), Geschlechterrollen und Frauenbild in der Perspektive antiker Autoren. Innsbruck etc.: Studien-Verlag, 13-55.

Bichler, Reinhold; Rollinger, Robert (2001) Herodot. 2. Aufl. Hildesheim etc.: Olms.

Birkhan, Helmut (1970) Germanen und Kelten bis zum Ausgang der Römerzeit: der Aussagewert von Wörtern und Sachen für die frühesten keltisch-germanischen Kulturbeziehungen. Wien: Verl. der Österr. Akad. der Wiss.

\footnotetext{
${ }^{29}$ Pohl 1988; Pohl \& Diesenberger 2002.

30 Scheibelreiter 1999.

31 Wolfram \& Schwarcz 1988; Chrysos \& Schwarz 1989.

32 Birkhan 1970.

${ }^{33}$ Birkhan 1999a und 1999b.
} 
Birkhan, Helmut (1999a) Kelten: Bilder ihrer Kultur. Wien: Verl. der Österr. Akad. der Wiss.

Birkhan, Helmut (1999b) Kelten: Versuch einer Gesamtdarstellung ihrer Kultur. 3. Aufl. Wien: Verl. der Österr. Akad. der Wiss.

Burian, Jan (1996) ,Rezension von: Dobesch 1995.' - Listy filologické 119, 235-237.

Chrysos, Evangelos K.; Schwarcz, Andreas (Hrsg.) (1989) Das Reich und die Barbaren. Wien etc: Böhlau. (Veröffentlichungen des Instituts für Österreichische Geschichtsforschung; 29.)

Daim, Falko; Andrási, Júlia (2000) Die Awaren am Rand der byzantinischen Welt: Studien zu Diplomatie, Handel und Technologietransfer im Frühmittelalter. Innsbruck: Univ.Verl. Wagner. (Monographien zur Frühgeschichte und Mittelalterarchäologie; 7.)

Daim, Falko; Bálint, Csanád (1989) Archäologie der Steppe: Steppenvölker zwischen Volga und Donau vom 6. bis zum 10. Jh. Wien: Böhlau.

Dobesch, Gerhard (1966) Caesars Apotheose zu Lebzeiten und sein Ringen um den Königstitel. Untersuchungen über Caesars Alleinherrschaft. Wien: Österreichisches Archäologisches Institut.

Dobesch, Gerhard (1977) ,Alte Geschichte an der Universität Wien seit 1876.' Hundert Jahre Institut für Alte Geschichte, Archäologie und Epigraphik der Universität Wien: 1876-1976. Wien: Institut für Alte Geschichte, Archäologie und Epigraphik, $14-24$.

Dobesch, Gerhard (1980) Die Kelten in Österreich nach den ältesten Berichten der Antike: das norische Königreich und seine Beziehungen zu Rom im 2. Jahrhundert v. Chr. Wien etc.: Böhlau.

Dobesch, Gerhard (1994) Vom äußeren Proletariat zum Kulturträger: ein Aspekt zur Rolle der Germanen in der Spätantike. Amsterdam: Hakkert. (Geographica Historica; 6.)

Dobesch, Gerhard (1995) Das europäische „Barbaricum" und die Zone der Mediterrankultur: ihre historische Wechselwirkung und das Geschichtsbild des Poseidonios. Wien: Holzhausen. (Tyche: Supplementband; 2.)

Dobesch, Gerhard (2004a) Evropské „barbaricum“ a zóna středomořské kultury: jejich vzájemné historické pưsobení a Poseidóniưv obraz dějin. České Budějovice: Historický Ústav Jihočeské Univerzity. (Relationes Budvicenses supplementa.)

Dobesch, Gerhard (2004b) Vita et bibliographia. I. Lisový et K. Tomaschitz edendum curaverunt. České Budějovice: Historický Ústav Jihočeské Univerzity; Wien: Wiener Humanistische Gesellschaft.

Dörpfeld, Wilhelm; Reisch, Emil (1896) Das griechische Theater: Beiträge zur Geschichte des Dionysos-Theaters in Athen und anderer griechischer Theater. Athen: Barth \& von Hirst.

Franke, Peter Robert (2001) ,Vorwort [G. Dobesch].' - Gerhard Dobesch, Ausgewählte Schriften. Bd. 1: Griechen und Römer. Hrsg. von H. Heftner und K. Tomaschitz. Köln etc.: Böhlau, XVI-XX.

Friesinger, Herwig; Adler, Horst (1979) Die Zeit der Völkerwanderung in Niederösterreich. St. Pölten; Wien: Verlag Niederösterreichisches Pressehaus. (Wissenschaftliche Schriftenreihe Niederösterreich; 41/42.)

Friesinger, Herwig (Hrsg.) (1994) Markomannenkriege: Ursachen und Wirkungen. 6. Internationales Symposium "Grundprobleme der Frühgeschichtlichen Entwicklung im Nörd- 
lichen Mitteldonaugebiet", Wien, 23.-26. November 1993. Brno: Archäologisches Institut der Akademie der Wissenschaften der Tschechischen Republik.

Friesinger, Herwig; Vacha, Brigitte (1987) Die vielen Väter Österreichs: Römer, Germanen, Slawen. Wien: Compress-Verlag.

Friesinger, Herwig; Vacha, Brigitte (1988) Römer - Germanen - Slawen in Österreich, Bayern und Mähren: eine Spurensuche. Stuttgart: Theiss.

Graßl, Herbert (1998) ,Zum frühgeschichtlichen Leben in den Alpen im Lichte antiker Literatur.' - Österreich in Geschichte und Literatur 42, 123-127.

Graßl, Herbert (2000) ,Die Taurisker: Beiträge zur Geschichte und Lokalisierung eines antiken Ethnonyms.' - Orbis terrarum 6, 127-138.

Graßl, Herbert (2001) ,Der Gründungsheros von Virunum.' - Carinthia Romana und die römische Welt: Festschrift für Gernot Piccottini zum 60. Geburtstag. Klagenfurt: Geschichtsverein für Kärnten, 25-32.

Haider, Peter (1993) ,Zu den „norischen Tauriskern“ - eine quellen- und literaturkritische Studie.' - Lippert, A. (Hrsg.), Hochalpine Altstraßen im Raum Bad Gastein-Mallnitz. Wien: VWGÖ, 219-247.

Haider, Peter (2000) "Historisches und Sprachgeschichtliches zu den „rätischen“ Inschriften am Schneidjoch bei Steinberg am Rofan.' - Hainzmann, M. (Hrsg.), VOTIS · XX · SOLVTIS. Jubiläumsschrift der Archäologischen Gesellschaft Steiermark. (AGST - Nachrichtenblatt 1-2, 1999.) Graz: Acad. Publ., 71-76.

Hainzmann, Manfred (1994) ,Die epigraphischen Zeugnisse für die boiischen Fürstennamen.' - Göbl, R. (Hrsg.), Die Hexadrachmenprägung der Groß-Boier. Wien: Fassbaender, 77-87.

Hainzmann, Manfred (1996) ,Die Kelten vom Magdalensberg. Eine onomastische Bestandsaufnahme.' - Jerem, Erzsébet (Hrsg.), Die Kelten in den Alpen und an der Donau: Akten des internationalen Symposions St. Pölten, 14.-18. Oktober 1992. Budapest etc: Wien: Archaeolingua Alapítvány, 447-459.

Hainzmann, Manfred (1998) ,Kelten in der Steiermark - zur Namenevidenz.' - Die Zeit der Kelten (Sonderausstellung im Volkshaus Bärnbach). Graz: Abteilung für Vorund Frühgeschichte und Münzensammlung am Landesmuseum Joanneum, 67-81.

Hainzmann, Manfred (2001a) ,Der Raum Drau-Mur-Raab aus epigraphischer Sicht. Zum einheimischen Namengut in römerzeitlichen Inschriften.' - Lippert, A. (Hrsg.), Die Drau-, Mur- und Raab-Region im 1. vorchristlichen Jahrtausend: Akten des internationalen und interdisziplinären Symposiums vom 26. bis 29. April 2000 in Bad Radkersburg. Bonn: Habelt, 335-341.

Hainzmann, Manfred (2001b) ,The F.E.R.C.AN. Project. Fontes epigraphici religionis Celticae antiquae.' - Zeitschrift für Celtische Philologie 52, 211-218.

Hainzmann, Manfred (2002) ,Taranis-Jupiter: Keltischer Donner und römischer Blitz.' - Ternes, Ch. M.; Zinser, H. (Hrsg.), Dieux des Celtes = Götter der Kelten = Gods of the Celts. Luxemburg: EURASSOC, 19-38.

Hofeneder, Andreas (2005) Die Religion der Kelten in den antiken literarischen Zeugnissen: Sammlung, Übersetzung und Kommentierung. Band I. Von den Anfängen bis Caesar. Wien: Verl. der Österreichischen Akad. der Wiss. (Mitteilungen der Prähistorischen Kommission; 59.)

Kenner, Hedwig (1977) ,Klassische Archäologie an der Universität Wien seit 1876.' Hundert Jahre Institut für Alte Geschichte, Archäologie und Epigraphik der Universität 
Wien: 1876-1976. Wien: Institut für Alte Geschichte, Archäologie und Epigraphik, 3-13.

Lisový, Igor; Tomaschitz, Kurt (2003-2004) ,Gerhard Dobesch.' - Relationes Budvicenses 4-5 (Ioanni Burian LXXV), 173-175.

Pohl, Walter (1988) Die Awaren: ein Steppenvolk in Mitteleuropa, 567-822 n. Chr. München: Beck.

Pohl, Walter; Diesenberger, Maximilian (Hrsg.) (2002) Integration und Herrschaft: ethnische Identitäten und soziale Organisation im Frühmittelalter. Wien: Verl. der Österr. Akad. der Wiss.

Reisch, Emil (1890) Griechische Weihgeschenke. Prag etc.: Tempsky etc. (Abhandlungen des archaeologisch-epigraphischen Seminars der Universität Wien; 8.)

Rollinger, Robert (1995), Zog Drusus auf seinem Weg durch die Alpen im Jahre 15 v. Chr. durch das Montafon? oder: Notizen zu einer ,Alten Geschichte' des Tales am Oberlauf der Ill.' - Rudigier, A.; Strasser, P. (Hrsg.), Montafon: Beiträge zur Geschichte und Gegenwart. Festschrift für Eleonore Schönborn zum 75. Geburtstag (= Bludenzer Geschichtsblätter, Heft 24-26), Bludenz: Geschichtsverein Region Bludenz, 213-230.

Rollinger, Robert (1998),Zum Alamannenfeldzug Constantius' II. an Bodensee und Rhein im Jahre $355 \mathrm{n}$. Chr. und zu Julians erstem Aufenthalt in Italien. Überlegungen zu Ammianus Marcellinus 15,4.' - Klio 80, 231-262.

Rollinger, Robert (2001) ,Raetiam autem et Vindelicos ac Noricos Pannoniamque et Scordiscos novas imperio nostro subiunxit provincias. Oder: Wann wurde Raetien (einschließlich Noricums und Pannoniens) als römische Provinz eingerichtet? Eine Studie zu Vell. 2,38f (mit einigen einleitenden Bemerkungen zur 'provinzialrömischen Geschichte' im wissenschaftlichen Oeuvre Franz Hampls).' - Haider, Peter W.; Rollinger, Robert (Hrsg.), Althistorische Studien im Spannungsfeld zwischen Universal- und Wissenschaftsgeschichte. Festschrift für Franz Hampl gedacht zum 90. Geburtstag am 8. Dezember 2000. Stuttgart: Steiner, 267-315.

Scheibelreiter, Georg (1999) Die barbarische Gesellschaft: Mentalitätsgeschichte der europäischen Achsenzeit 5.-8. Jh. Darmstadt: WB.

Schober, Arnold (1923) Die römischen Grabsteine von Noricum und Pannonien. Wien: Hölzel. (Sonderschriften des Österreichischen Archöologischen Institutes in Wien; 10.)

Schober, Arnold (1951) Die Kunst von Pergamon. Wien etc.: Rohrer.

Sieberer, Wido (1995) Das Bild Europas in den Historien: Studien zu Herodots Geographie und Ethnographie Europas und seiner Schilderung der persischen Feldzüge. Innsbruck: Inst. für Sprachwiss. der Univ. (Innsbrucker Beiträge zur Kulturwissenschaft: Sonderheft; 96.)

Strobel, Karl (1984) Untersuchungen zu den Dakerkriegen Trajans: Studien zur Geschichte des mittleren und unteren Donauraumes in der Hohen Kaiserzeit. Bonn: Habelt.

Strobel, Karl (1989) Die Donaukriege Domitians. Bonn: Habelt. (Antiquitas: Reihe 1, Abhandlungen zur alten Geschichte; 38.)

Strobel, Karl (1996) Die Galater: Geschichte und Eigenart der keltischen Staatenbildung auf dem Boden des hellenistischen Kleinasien. Berlin: Akademie-Verlag.

Strobel, Karl (2003) ,Die Noreia-Frage: neue Aspekte und Überlegungen zu einem alten Problem der historischen Geographie Kärntens.' - Carinthia I 193, $25-71$. 
Stuppner, Alois (1994), Zu den Auswirkungen der Markomannenkriege im nördlichen Niederösterreich.' - Friesinger, Herwig (Hrsg.) (1994) Markomannenkriege: Ursachen und Wirkungen. 6. Internationales Symposium "Grundprobleme der Frühgeschichtlichen Entwicklung im Nördlichen Mitteldonaugebiet", Wien, 23.-26. November 1993. Brno: Archäologisches Institut der Akademie der Wissenschaften der Tschechischen Republik, 285-298.

Stuppner, Alois (1995) ,Einige Bemerkungen zu den frühkaiserzeitlichen, römischgermanischen Handelsbeziehungen im nördlichen Niederösterreich.' - Tejral, J. (Hrsg.), Kelten, Germanen, Römer im Mitteldonaugebiet: vom Ausklang der LatèneZivilisation bis zum 2. Jahrhundert. Brno: Archeologický Ústav (Nitra), 199-215.

Stuppner, Alois (1997) ,Römer und Germanen an der mittleren Donau.' - Friesinger, H.; Krinzinger, F. (Hrsg.), Der römische Limes in Österreich. Wien: Verl. der Österr. Akad. der Wiss., 113-127.

Tausend, Klaus (1984) Die Darstellung der Hunnen bei Ammianus Marcellinus, Priskos, Iordanes und den lateinischen Dichtern und Panegyrikern. Diss. Graz.

Tausend, Klaus (1985/86) ,Hunnische Poliorketik.' - Grazer Beiträge 12/13, 265-281.

Tausend, Klaus (1987) ,Die Bedeutung des Importes aus Germanien für den römischen Markt.' - Tyche 2, 217-227.

Tausend, Klaus (1988) ,Caesars germanische Reiter.' - Historia 37, 491-497.

Tausend, Klaus (1991) ,Zur politischen Rolle germanischer Kultverbände.' - Historia 40, 248-257.

Tausend, Klaus (1997) ,Lugier - Wandilier - Wandalen.' - Tyche 12, 229-236.

Tausend, Klaus (1999a) ,Bemerkungen zum Wandaleneinfall des Jahres 270.' Historia 48, 119-127.

Tausend, Klaus (1999b) ,Inder in Germanien.' - Orbis Terrarum 5, 115-125.

Tomaschitz, Kurt (2002) Die Wanderungen der Kelten in der antiken literarischen Überlieferung. Wien: Verl. der Österreichischen Akad. der Wiss. (Mitteilungen der Prähistorischen Kommission; 47.)

Urban, Otto H. (1994) Keltische Höhensiedlungen an der mittleren Donau. Bd. 1: Der Freinberg. Linz: Stadtmuseum Nordico. (Linzer Archäologische Forschungen; 22.)

Urban, Otto H. (1995) Keltische Höhensiedlungen an der mittleren Donau. Bd. 2: Der Braunsberg. Linz: Stadtmuseum Nordico. (Linzer Archäologische Forschungen; 23.)

Urban, Otto H. (2003) Der lange Weg zur Geschichte: die Urgeschichte Österreichs. Wien: Ueberreuter. [Erstmals erschienen in 2000.]

Wolfram, Herwig (1987) Die Geburt Mitteleuropas: Geschichte Österreichs vor seiner Entstehung, 378-907. Berlin: Siedler.

Wolfram, Herwig (1990) Das Reich und die Germanen: zwischen Antike und Mittelalter. Berlin: Siedler.

Wolfram, Herwig (2001) Die Goten: von den Anfüngen bis zur Mitte des sechsten Jahrhunderts. Entwurf einer historischen Ethnographie. 4. Aufl. München: Beck. (1. Aufl. 1979 unter dem Titel Geschichte der Goten: Entwurf einer historischen Ethnographie.)

Wolfram, Herwig; Daim, Falko (Hrsg.) (1980) Die Völker an der mittleren und unteren Donau im fünften und sechsten Jahrhundert. Berichte des Symposions der Kommission für Frühmittelalterforschung, 24. bis 27. Oktober 1978, Stift Zwettl, Niederösterreich. Wien: Verl. der Österr. Akad. der Wiss. 
Wolfram, Herwig; Friesinger, Herwig (Hrsg.) (1985) Die Bayern und ihre Nachbarn. Berichte des Symposions der Kommission für Frühmittelalterforschung, 25. bis 28. Oktober 1982, Stift Zwettl, Niederösterreich. Wien: Verl. der Österr. Akad. der Wiss.

Wolfram, Herwig; Schwarcz, Andreas (Hrsg.) (1988) Anerkennung und Integration: zu den wirtschaftlichen Grundlagen der Völkerwanderungszeit 400-600. Berichte des Symposions der Kommission für Frühmittelalterforschung, 7. bis 9. Mai 1986 Stift Zwettl, Niederösterreich. Wien: Verl. der Österr. Akad. der Wiss. 